\title{
An evidence based approach to testing for antibody to herpes simplex virus type 2
}

\author{
A J Copas, F M Cowan, A L Cunningham, A Mindel
}

See end of article for authors' affiliations

.....................

Correspondence to: Dr Andrew Copas, Department of Sexually Transmitted Diseases, Royal Free and University College London Medical School,

Mortimer Market Centre,

Off Capper Street, London WCIE 6AU, UK;

acopas@gum.ucl.ac.uk

Accepted for publication 28 June 2002
Sex Transm Infect 2002; 78:430-434

\begin{abstract}
Objectives: To establish whether a simple risk scoring system, based on limited information, can reflect the variation in HSV-2 prevalence in a population, and whether a common system can be used across settings. To establish whether knowledge of a patient's score can aid the interpretation of the result from one of the commercial type specific assays.

Methods: Four previous cross sectional studies are considered, with HSV-2 antibody results by western blot or type specific ELISA tests. The clinical settings were a blood donor centre (1359 participants) and STD clinic (808 participants), London, United Kingdom, an antenatal clinic, Sydney, Australia (2317 participants), and a family medical centre, Seattle, United States (478 participants). We determined the factors associated with HSV-2 prevalence, the similarity of associations across settings, and the variation in HSV-2 prevalence by risk score.

Results: A simple scoring captured much variation in HSV-2 prevalence in each population-for example, for London blood donors, scoring based on sex, age, and number of lifetime partners, prevalence varied from $0.7 \%(95 \% \mathrm{Cl} 0.1$ to 2.0$)$ to $47.3 \%(37.9$ to 56.6$)$ across five risk groups. For number of lifetime partners, and sex, the association with HSV-2 varied significantly across studies. Conclusions: A scoring system can aid test interpretation-for example, in London blood donors the post-test probability of infection following a positive result varies from around $25 \%$ to $98 \%$ across risk groups for a typical type specific assay. Further work could address whether this theoretical benefit can be realised in practice. A common risk scoring probably could not be used across settings.
\end{abstract}

$\mathrm{H}$ erpes simplex virus type 2 (HSV-2) is the predominant cause of recurrent genital herpes. Seroepidemiological studies from the United States and elsewhere indicate that most people who are infected with HSV-2 are unaware of their infection. ${ }^{1}$ What is more, two thirds of people presenting with their first episode of genital herpes turn out to have been infected by a sexual partner with previously unrecognised genital herpes. ${ }^{2}$ Recently, assays which can reliably identify type specific antibody to HSV-2 have become commercially available. These assays can be used to diagnose HSV-2 infection among asymptomatic but, none the less, infected people who would otherwise be unaware of their infection. However, type specific assays for antibody to HSV-2 are imperfect, in that their specificity and sensitivity are not $100 \% .{ }^{3}$ Use of these tests will result in some uninfected individuals being falsely diagnosed as being infected with HSV-2 (false positive result). Conversely, some infected individuals may have a negative HSV-2 antibody test result and be falsely reassured that they are uninfected (false negative result). As genital herpes is a lifelong sexually transmitted infection, which may be transmitted to sexual partners in the absence of symptoms, either a false negative or false positive diagnosis could have serious consequences for that individual and their current and future sexual partners.

The positive predictive value (or post-test probability of being infected) of an HSV-2 antibody test is the probability of an individual being truly positive given a positive result. The negative predictive value (or post-test probability of being uninfected) is the probability of being truly negative given a negative result. These predictive values of the test depend on the test characteristics and also the individual's risk of infection (or pretest probability). The local population prevalence can be used to estimate an individual's risk. However the prevalence of HSV-2 varies widely both within and between populations by age, sex, and past sexual behaviour, ${ }^{4-7}$ so it is important to include such factors in any measure of risk.
In this study we wanted to find out whether it is possible to estimate the pretest probability using a simple risk score, based on demographic, clinical, and behavioural information that is routinely collected when taking a sexual history. This could then be used by physicians to help interpret the HSV-2 antibody test result as advocated by Jaeschke et $\mathrm{al}^{8}$ and, in particular, to determine the likelihood that the test result is true or false. That is by combining the pretest probability with the likelihood ratio of the assay in order to establish the post-test probability of infection. The risk scoring system could even be used to develop an evidence based testing strategy. We also consider whether it is likely that it would be possible to use the same risk scoring system in four different populations.

\section{METHODS}

We used data collected by self completion questionnaire from four populations, in three countries (United Kingdom, Australia, and United States) as part of seroepidemiological studies of HSV-2 (see table 1). Further details of these studies are given elsewhere. ${ }^{4}{ }^{10}$

\section{Developing a risk score for each study}

We used standard univariate statistical tests to identify sociodemographic, behavioural, and clinical factors significantly associated with antibody to HSV-2. Forwards stepwise selection was then used to select the best of these factors to form a logistic regression model for HSV-2. The log odds ratios of the factors in the model are taken to indicate their importance, so that an additive scoring system results. The value of one unit of risk on the log odds scale was then selected so that as many factors as possible were close to a whole number of units and no factor represented more than five units. Each factor was then assigned a number of risk units, equal to the log odds ratio divided by the unit value, rounded to the nearest whole number. The overall risk score is then the sum of these terms. 
Table 1 The four studies

\begin{tabular}{lllll}
\hline Study No & Location, and year & Patient setting & Diagnostic test used & $\begin{array}{l}\text { Number of patients with full } \\
\text { risk information }\end{array}$ \\
\hline 1 & London, 1992 & Blood donors & Modified western blot & 1359 \\
2 & London, 1990-2 & STI clinic attendees & Modified western blot & 808 \\
3 & Sydney, 1995-8 & Women, antenatal care & Type specific ELISA & 2317 \\
4 & Seattle, 1991-3 & Family medical centre & Western blot & 476 \\
\hline
\end{tabular}

For example, in study 4, one unit of risk was chosen to be 0.65 on the log odds scale. For number of lifetime sexual partners, relative to $0-2$ the $\log$ odds ratios for 3-4, 5-9, and $10+$ were $1.43,2.10$, and 2.80 respectively. Dividing by 0.65 and rounding, we see that $3-4,5-9$, or $10+$ partners represent 2,3 , or 4 units of risk respectively.

The sample HSV prevalence is calculated for each value of the risk score. The score is then grouped to capture as much of the variation in prevalence as possible, subject to no more than five groups in total and each containing at least $8 \%$ of the sample to allow accurate estimation of the prevalence. However, for study 3, owing to similar prevalence across the middle risk scores, only four groups are created of which the highest risk group contains only $4 \%$ of the sample but has a substantially higher prevalence. For each risk group the sample prevalence and $95 \%$ confidence interval are determined.

\section{Assessing similarity of associations with HSV-2 across settings}

To consider whether a common scoring system could be used in different settings around the world, we assessed the similarity of associations with HSV-2 in the different studies. Age, sex, and number of lifetime partners were collected in each study and associated with HSV-2. Odds ratios for these factors, adjusting for the other two, were calculated using logistic regression. The similarity of the associations (that is, odds ratios) across studies can be formally assessed by testing for the main effects of study and interactions with the other factors.

All analysis was performed in SPSS 10 Or STATA 6.

\section{RESULTS}

\section{Developing the risk scores}

We include detailed results for study 1 in order to illustrate how the algorithm was constructed; results for other studies are available from the authors on request. Sex, age, genital blisters, lifetime number of partners, history of non-specific urethritis (NSU), gonococcus, warts, lice, Trichomonas vaginalis (TV), and thrush are all significantly associated with HSV-2. Country of birth, ethnicity, marital status, oral blisters/sores, cold sores, mouth ulcers, sexuality, age at first sex are not. Table 2 indicates the factors identified in the model selection process. The interactions between sex and the other three terms were each tested, and none found to significantly add to the model. The score to be added for each factor is specified in the final column of table 2. For example, if the patient is a 23 year old ( 0 points) man ( 0 points $)$ with three lifetime partners ( 2 points) and no history of genital blisters ( 0 points) his score is 2 , whereas if the patient is a 34 year old ( 2 points) woman ( 2 points) with seven lifetime partners ( 2 points) who has never had genital blisters ( 0 points) her score is 6 .

The resulting scores, ranging from $0-10$, were grouped according to the HSV-2 prevalence into five levels: $0-3,4,5,6$, and 7 or more. In table 3 the prevalence of antibody to HSV-2 is presented by risk group. In study 1 , for example, the prevalence increases dramatically from $0.7 \%$ to $46.3 \%$ across the risk groups. The corresponding figures are also presented for the other studies.

\begin{tabular}{lll}
$\begin{array}{l}\text { Table } 2 \\
\text { scoring for study } 1\end{array}$ & \multicolumn{2}{l}{ Best fitting model and associated risk } \\
\hline Factor & OR $(95 \% \mathrm{Cl})$ & Add to score \\
\hline Sex & & \\
Male & $1-$ & 0 \\
Female & $9.81(5.35$ to 18.0$)$ & 2 \\
Age (years) & & \\
24 or less & $1-$ & 0 \\
$25-29$ & $2.90(0.87$ to 9.69$)$ & 1 \\
$30+$ & $8.78(2.88$ to 26.7$)$ & 2 \\
Number of lifetime sexual partners & 0 \\
0 & $1-$ & 0 \\
$1-2$ & $1.16(0.12$ to 11.1$)$ & 0 \\
$3-9$ & $5.14(0.63$ to 41.8$)$ & 2 \\
$10+$ & $18.63(2.27$ to 153$)$ & 3 \\
Genital blisters & $1-$ & 0 \\
Never & $5.93(1.33$ to 26.4$)$ & 2 \\
Once & $16.36(5.56$ to 48.1$)$ & 3 \\
Less than once per year & $18.50(7.05$ to 48.6$)$ & 3 \\
$1-3$ times per year & $20.34(4.11$ to 101$)$ & 3 \\
$4+$ times per year &
\end{tabular}

\section{Assessing similarity of associations with HSV-2 across settings}

After controlling for the three risk factors, studies 2 and 4 have significantly higher HSV-2 prevalence than the other studies. Relative to study 1, the study odds ratios (95\% CI) are 3.18 (2.38 to 4.26$), 1.82$ ( 1.39 to 2.37 ), and 3.20 (2.39 to 4.29 ) for studies 2, 3, and 4 .

Furthermore we found the associations with $\operatorname{sex}\left(\chi^{2}=15.6\right.$, $2 \mathrm{df}, \mathrm{p}=0.0004)$, and number of partners $\left(\chi^{2}=17.5,6 \mathrm{df}, \mathrm{p}=\right.$ $0.008)$, differed significantly across studies. Table 4 shows that, relative to study one, in study 2 the association between sex and HSV-2 is weaker, and in study 3 the association between number of partners and HSV-2 is weaker. The association with age did not vary significantly $\left(\chi^{2}=8.6,6 \mathrm{df}, \mathrm{p}=0.20\right)$ across studies.

\section{CONCLUSIONS AND FURTHER WORK}

We have seen that it is possible to develop a risk score, which helps to predict an individual's risk of HSV-2 on the basis of only a few pieces of information in all four study settings. These data will usually be gathered routinely during sexual history taking. Each of the resulting risk groups contains an appreciable proportion of people tested, yet the prevalence of HSV-2 varies sufficiently across groups in each study to have a substantial impact on the post-test probabilities of infection (following either a positive or negative test), and hence test interpretation. This is illustrated in table 5, where three realistic tests are considered. ${ }^{3}$ For example, someone with a low risk score should be greatly reassured by a negative test result as their post-test probability of infection is also low. If they have a positive result, however, they should be aware that this has a significant likelihood of being false, as their post-test probability will be substantially less than $100 \%$, even using a test with $98 \%$ sensitivity and specificity. The converse is true 


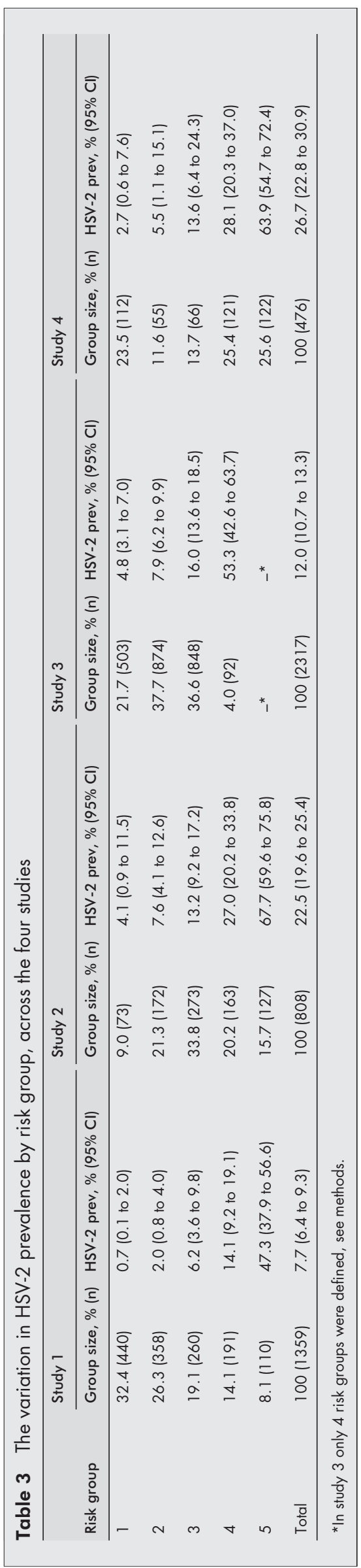

\section{Key messages}

- Four patient databases of HSV-2 test results, demographic, clinical, and behavioural information from settings in London, Seattle, and Sydney were analysed

- In each setting a simple risk scoring system, based on limited information, successfully reflected much of the variation in HSV-2 prevalence

- Where commercial type specific assays are used, such a risk scoring system could be used in combination with the test result, to inform physician and patient of the likelihood of infection

- Further work could address whether the use of such risk scoring is feasible and beneficial in practice.

for individuals at high risk of infection. In addition to informing test interpretation, the patient's pretest probability can be used in advance of testing. For example, where it is clear that a patient's post-test probability will be inconclusive (for example, $20 \%-80 \%$ ) whether the test result is positive or negative, then the clinician and patient can discuss whether the test is likely to be of benefit or not. At present many physicians are not offering testing for HSV-2 antibody on the basis that they may do more harm than good (for example, by erroneously telling someone that they have a lifelong, viral, sexually transmitted infection). By using a simple algorithm such as the ones that are outlined here, preferably in conjunction with the patient, it is less likely that false results will be given. Additionally it may help the patient understand the limitations of testing in certain circumstances.

Alternatively, in those few laboratories able to offer confirmatory HSV-2 antibody testing using a western blot assay, this approach could be used to decide which samples to retest using western blot. (The HSV-2 antibody western blot assay is expensive and labour intensive and unlikely to become widely or commercially available. ${ }^{3}$ )

We have not evaluated this approach for HSV-1 testing as we feel this is a less controversial area. The overall prevalence of HSV-1 infection in the community is higher so there is less likelihood of false positive and negative diagnoses. Additionally, the implications of either a false positive or negative diagnosis are likely to be less important. HSV-1 is generally considered a minor, viral illness and even if genitally acquired is unlikely to lead to recurrent genital herpes.

Since the broad themes of sexual behaviour, age, sex, and clinical symptoms are associated with HSV-2 prevalence in every setting it is tempting to feel that a common risk scoring can be developed and applied identically at different sites. This would certainly be of greatest practical use. It is true that if the risk score from one setting is applied to another then it is likely to describe a substantial amount of the variation in the second setting. For example, we applied the risk scoring from study 1 to individuals in study 2 and found that the prevalence in the risk groups increased from $1.4 \%$ to $50.7 \%$.

However, our findings that three key behavioural and demographic risk factors have significantly different associations across studies, reflecting the different make up of the populations concerned, and that study itself is a risk factor, suggest that a common scoring system based on such factors is not possible. In general some individuals will be assigned to inappropriate risk groups-for example, those with a major risk factor in one setting which is a mild risk factor in another. In addition, it is not possible to say exactly what the prevalence will be in the risk groups when a scoring based on one setting is applied to another. This finding is perhaps not surprising, firstly, because it is impossible to measure the exact behavioural risk experienced by an individual since it requires information unlikely to be available to the patient-for example, detailed risk information for each lifetime partner. Secondly, a related problem is that the association between 
Table 4 Assessing comparability of HSV-2 risk associations across studies for three risk factors

\begin{tabular}{|c|c|c|c|c|}
\hline Factor & Study 1 & Study 2 & Study 3 & Study 4 \\
\hline \multicolumn{5}{|c|}{ Lifetime sexual partners } \\
\hline $0-2$ & $1-$ & $1-$ & $1-$ & $1-$ \\
\hline $3-9$ & 6.01 (2.52 to 14.3$)$ & $4.27(0.97$ to 18.8$)$ & 2.26 (1.71 to 2.98$)$ & 7.99 (2.77 to 23.0 ) \\
\hline $10+$ & $20.3(8.40$ to 49.0$)$ & 7.18 (1.66 to 31.1$)$ & 3.84 (2.74 to 5.38$)$ & $19.8(6.87$ to 57.0$)$ \\
\hline \multicolumn{5}{|c|}{ Age (years) } \\
\hline$\leqslant 29$ & $1-$ & $1-$ & $1-$ & $1-$ \\
\hline $30-34$ & 2.96 (1.50 to 5.82$)$ & 2.73 (1.75 to 4.24$)$ & 1.65 (1.24 to 2.18$)$ & $1.65(0.88$ to 3.10$)$ \\
\hline $35+$ & $4.22(2.47$ to 7.20$)$ & $3.96(2.52$ to 6.23$)$ & $2.46(1.73$ to 3.48$)$ & $2.34(1.41$ to 3.90$)$ \\
\hline \multicolumn{5}{|c|}{0.10 [2.02 100.201} \\
\hline Male & $1-$ & $1-$ & Women only & $1-$ \\
\hline Female & 7.82 (4.59 to 13.3$)$ & 2.14 (1.46 to 3.14$)$ & & 5.55 (3.29 to 9.36$)$ \\
\hline
\end{tabular}

Table 5 Post-test probability of infection for those at lowest and highest risk, under three hypothetical diagnostic tests*

\begin{tabular}{|c|c|c|c|c|c|c|c|c|}
\hline \multirow[b]{2}{*}{ Study } & \multirow[b]{2}{*}{ Risk group } & \multirow{2}{*}{$\begin{array}{l}\text { Pretest probability of } \\
\text { infection, \% }\end{array}$} & \multicolumn{3}{|c|}{$\begin{array}{l}\text { Probability of infection following a positive } \\
\text { test, \% }\end{array}$} & \multicolumn{3}{|c|}{$\begin{array}{l}\text { Probability of infection following a negative } \\
\text { test result, \% }\end{array}$} \\
\hline & & & Test 1 & Test 2 & Test 3 & Test 1 & Test 2 & Test 3 \\
\hline 1 & $\begin{array}{l}\text { Lowest } \\
\text { Highest }\end{array}$ & $\begin{array}{l}0.7 \\
47.3\end{array}$ & $\begin{array}{l}24.5 \\
97.6\end{array}$ & $\begin{array}{l}25.1 \\
97.7\end{array}$ & $\begin{array}{l}25.7 \\
97.8\end{array}$ & $\begin{array}{l}0.06 \\
6.8\end{array}$ & $\begin{array}{l}0.04 \\
4.4\end{array}$ & $\begin{array}{l}0.01 \\
1.8\end{array}$ \\
\hline 2 & $\begin{array}{l}\text { Lowest } \\
\text { Highest }\end{array}$ & $\begin{array}{l}4.1 \\
67.7\end{array}$ & $\begin{array}{l}66.3 \\
99.0\end{array}$ & $\begin{array}{l}67.0 \\
99.0\end{array}$ & $\begin{array}{l}67.7 \\
99.0\end{array}$ & $\begin{array}{l}0.34 \\
14.6\end{array}$ & $\begin{array}{l}0.22 \\
9.7\end{array}$ & $\begin{array}{l}0.09 \\
4.1\end{array}$ \\
\hline 3 & $\begin{array}{l}\text { Lowest } \\
\text { Highest }\end{array}$ & $\begin{array}{l}4.8 \\
53.3\end{array}$ & $\begin{array}{l}69.9 \\
98.1\end{array}$ & $\begin{array}{l}70.5 \\
98.2\end{array}$ & $\begin{array}{l}71.2 \\
98.2\end{array}$ & $\begin{array}{l}0.41 \\
8.5\end{array}$ & $\begin{array}{l}0.26 \\
5.5\end{array}$ & $\begin{array}{l}0.10 \\
2.3\end{array}$ \\
\hline 4 & $\begin{array}{l}\text { Lowest } \\
\text { Highest }\end{array}$ & $\begin{array}{l}2.7 \\
63.9\end{array}$ & $\begin{array}{l}56.1 \\
98.8\end{array}$ & $\begin{array}{l}56.9 \\
98.8\end{array}$ & $\begin{array}{l}57.6 \\
98.9\end{array}$ & $\begin{array}{l}0.23 \\
12.6\end{array}$ & $\begin{array}{l}0.14 \\
8.3\end{array}$ & $\begin{array}{l}0.06 \\
3.5\end{array}$ \\
\hline
\end{tabular}

*The sensitivity/specificity (\%) of tests 1,2 , and 3 are $92 / 98,95 / 98$, and $98 / 98$ respectively.

sexual behaviour and HSV-2 prevalence is dependent on the prevalence in the population, which will vary across settings.

This work to assess whether a common risk scoring can be used across settings is limited by a lack of common patient information across studies, few clinical data collected, and the small number of studies included. In particular, a greater degree of commonality might be expected if the scoring includes a variety of clinical factors. Further projects might be designed without these limitations.

Further work might also address whether more complex approaches to selecting risk factors and combining this information into a scoring system would provide additional benefit. More complex approaches would be feasible, in particular, if the calculation of pretest and post-test probabilities is computerised.

Importantly, further work is also needed to establish whether using such an evidence based approach to HSV-2 testing such as the one described would actually benefit patients and clinicians in practice.

\section{ACKNOWLEDGEMENTS}

We thank Anna Wald, University of Washington for the Seattle study data and Dr Danielle Mercey and Dr Gilly Arthur for help with the manuscript.

The UK study was funded by the Wellcome Trust. The Australian study was funded by grants from the Ramaciotti Foundation and the Australian National Health and Medical Research Council.

Funding: This analysis of the four studies was funded by GlaxoSmithKline Research and Development.

Competing interests: AJC has received reimbursement for attending a conference from GlaxoWellcome. FC has received reimbursement for speaking and attending conferences from GlaxoWellcome and has acted as a consultant for them. AM and ALC have received reimbursement for speaking and attending conferences from GlaxoWellcome and SmithKline Beecham.

\section{CONTRIBUTORS}

AJC selected the statistical methodology and performed the statistical analysis; FC had the original idea for the study and provided the data from London; AJC and FC wrote the paper together; AM and ALC provided the data from Sydney and commented on the paper; AJC will act as guarantor for this paper.

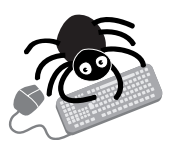

The full text of the questionnaires used in this study can be accessed online at www.sextransinf.com

Authors' affiliations

A J Copas, F M Cowan, Department of Sexually Transmitted Diseases, Royal Free and University College Medical School, Mortimer Market Centre, off Capper Street, London WC1E 6AU, UK

A L Cunningham, A Mindel, University of Sydney and Westmead Hospital, Sydney, Australia

\section{REFERENCES}

1 Corey L, Handsfield HH. Genital herpes and public health-addressing a global problem. JAMA 2000;283:791-4.

2 Mertz GJ, Schmidt O, Jourden JL, et al. Frequency of acquisition of first-episode genital infection with herpes simplex virus from symptomatic and asymptomatic source contracts. Sex Transm Dis 1985;12:33-9.

3 Ashley RL. Sorting out the new HSV type specific antibody tests. Sex Transm Infect 2001;77:232-7.

4 Cowan FM, Johnson AM, Ashley R, et al. Antibody to herpes simplex virus type 2 as a serological marker of sexual lifestyle in populations. BM 1994;309:1325-9. 
5 Nahmias AJ, Lee FK, Beckman-Nahmias S. Seroepidemiologic and sero-sociological patterns of Herpes simplex virus infection in the world. Scand J Infect Dis 1990;(Suppl 69): 19-36.

6 Fleming DT, McQullian GM, Johnson RE, et al. Herpes simplex virus type 2 in the United States, 1976 to 1994. N Engl J Med 1997:337: 1 105-11.

7 Obasi A, Mosha F, Quigley M, et al. Antibody to herpes simplex virus type 2 as a marker of sexual risk behaviour in rural Tanzania. J Infect Dis 1999;179:16-24.
8 Jaeschke R, Guyatt GH, Sackett DL, and for the Evidence Based Medicine Working Group. User's guide to the medical literature: III How Medicine Working Group. User's guide to the medical results and will to use an article about a diagnostic test $\mathrm{B}$. What are the results and
they help me in caring for my patients. JAMA 1994;271:703-7.

9 Wald A, Koutsky L, Ashley R, Corey L. Genital herpes in a primary care clinic: demographic and sexual correlates of herpes simplex type 2 infection. Sex Transm Dis 1997;24:149-55.

10 Mindel A, Taylor J, Tideman RL, et al. Neonatal herpes prevention: a minor public health problem in some communities. Sex Transm Infect 2000;76:287-91.

\section{ELECTRONIC PAGES}

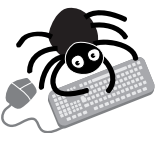

Please visit the Sexually Transmitted nfections website [www. sextransinf com] for links to the full articles

\section{Global views}

Sexually Transmitted Infections receives an increasing number of articles relating to prevalence of STIs or the performance of various syndromic management protocols in different populations. While these are very important for policymakers and clinicians locally, they tend to have limited applicability to other populations. For this reason we will publish these articles, after peer review, in full through eSTI. The paper edition of the journal will feature full abstracts in the "global view" section.

\section{Clinical characteristics of Chlamydia trachomatis infections in a general outpatient department of obstetrics and gynaecology in the Netherlands}

\section{J Bax, P M Oostvogel, J A E M Mutsaers, R Brand, M Craandiijk, J B Trimbos, P J Dörr}

Objective: Evaluation of prevalence and risk factors of Chlamydia trachomatis infections in an outpatient obstetric and gynaecological population.

Methods: A prospective, observational study was performed at an inner city hospital in The Hague, Netherlands. 1368 women attending the outpatient department of obstetrics and gynaecology participated in the study. For detection of $C$ trachomatis infections we used amplification of CT rRNA in urine samples (Gen Probe/AMPLIFIED-CT) and DNA probe for detection of CT rRNA from a urethral, endocervical, and anal swab (Gen Probe/PACE 2).

Results: The overall prevalence of $C$ trachomatis infections in our general obstetric and gynaecological population was $4.5 \%$. The prevalence in women under 30 years of age was $8.1 \%$. We found age and postcoital bleeding to be significant risk factors. We did not find significant differences between women from different ethnic origin or between women using different kinds of contraceptives. 12 (19.4\%) patients with $C$ trachomatis infections were found positive by urine test only, and $15(24.2 \%)$ only by DNA probe. Conclusions: Age is the most important risk factor in our population (overall prevalence $4.5 \%$, prevalence in women under 20 years of age $15.8 \%$ ). Analyses of urine and of endocervical specimens are complementary for the determination of the prevalence of $C$ trachomatis infections in women. Cost effectiveness analysis is needed to determine to what extent age based screening and/or antibiotic prophylaxis before intrauterine manipulations is indicated.

- (Sex Transm Infect 2002;78:e6) www.sextransinf.com/cgi/content/full/78/6/e6

Human T lymphotrophic virus-l (HTLV-I) infection in patients with unclassifiable dermatitis in central Kerala, south India: a preliminary study

\section{K Ajithkumar, S Ramalingam, R Kannangai, K J Prakash}

Objective: We have conducted a preliminary serostudy to confirm the presence of this virus in cases of dermatitis of unknown aetiology and among individuals with sexually transmitted infections (STI) in central Kerala.

Methods: 45 consecutive patients who attended the dermatology clinic of Medical College Kottayam with extensive dermatitis that could not be clinically classified into any known clinical entity and 37 consecutive patients who presented to the sexually transmitted disease (STD) clinic were enrolled for the study. Serum/plasma samples were screened for anti-HTLV-I antibody. Reactive and indeterminate samples were confirmed by an immunoblot.

Results: Among 37 STD clinic attendants, none had antibody to HTLV-I while two individuals $(4.44 \%)$ among the 45 with dermatitis had antibody to HTLV-I.

Conclusions: Our study proves the presence of HTLV-I in a subset of individuals with poorly defined dermatitis in Kerala. Further larger studies are necessary to assess the extent of this problem and its relation to STI in Kerala.

\ (Sex Transm Infect 2002;78:e7) www.sextransinf.com/cgi/content/full/78/6/e7 